\title{
Smaller Deborah number inducing more serrated plastic flow of metallic glass
}

\author{
M.Q. Jiang ${ }^{\text {a }}$, S.Y. Jiang ${ }^{\text {a }}$, Z. Ling ${ }^{\text {a }}$ L.H. Dai ${ }^{\mathrm{a}, \mathrm{b}, *}$ \\ a State Key Laboratory of Nonlinear Mechanics, Institute of Mechanics, Chinese Academy of Sciences, Beijing 100190, PR China \\ ${ }^{\mathrm{b}}$ State Key Laboratory of Explosion Science and Technology, Beijing Institute of Technology, Beijing 100081, PR China
}

\section{A R T I C L E I N F O}

\section{Article history:}

Received 20 October 2008

Received in revised form 18 January 2009

Accepted 4 April 2009

Available online 9 May 2009

\section{PACS:}

61.43.Dq

62.20.F-

64.70.pe

71.15.Pd

Keywords:

Metallic glasses

Indentation

Flow serration

Simulation

Deborah number

\begin{abstract}
A B S T R A C T
Spherical nano-indentations of $\mathrm{Cu}_{46} \mathrm{Zr}_{54}$ bulk metallic glass (BMG) model systems were performed using molecular dynamics (MD) computer simulations, focusing specifically on the physical origin of serrated plastic flow. The results demonstrate that there is a direct correlation between macroscopic flow serration and underlying irreversible rearrangement of atoms, which is strongly dependent on the loading (strain) rate and the temperature. The serrated plastic flow is, therefore, determined by the magnitude of such irreversible rearrangement that is inhomogeneous temporally. A dimensionless Deborah number is introduced to characterize the effects of strain rate and temperature on serrations. Our simulations are shown to compare favorably with the available experimental observations.
\end{abstract}

(c) 2009 Elsevier B.V. All rights reserved.

\section{Introduction}

Bulk metallic glasses (BMGs), due to their unique amorphous structure [1,2], display complex flow properties [3-6], intermediate between liquid and solid, which is at the root of their potential applications [7-9]. Nanoindentation on BMGs is a key method for studying their flow behaviors, during which the serrated plastic flow in load-displacement $(\mathrm{p}-\mathrm{h})$ curves has been widely observed [10-20]. Such flow serration, strongly relating strain rate and ambient temperature, can be conspicuous, weak, and even disappears. Understanding the flow phenomenon, from the view point of spatially (shear-banding), temporally or spatiotemporally inhomogeneity, is well documented [11-13,16-21], yet still remains a puzzle to a large extent. Real-time experimental observations in situ on the flow are therefore necessary, but very difficult, due to the limit of spatial and temporal resolution [21-23]. Recently, the molecular dynamics (MD) simulation has been proposed as an effective way in modeling indentation on BMGs [24-26], pro-

\footnotetext{
* Corresponding author. Address: State Key Laboratory of Nonlinear Mechanics, Institute of Mechanics, Chinese Academy of Sciences, Beijing 100190, PR China. Tel.: +8610 82543958; fax: +861082543977.

E-mail address: lhdai@Inm.imech.ac.cn (L.H. Dai).
}

viding an in situ observation on atomic motions. Remarkable progress in this aspect has been achieved by Falk, Langer, Shi et al. $[25,26]$ by developing Argon's concept of shear transformation zones (STZs) [5]. They found that the load drops in a p-h curve, i.e. serrated plastic flow, correspond closely to the bursts in deformation activity associated with shear bands [25]. Spatially, the suppression of wing-like shear bands of post-deformed specimen at higher strain rate leads to milder serrations [26]. Naturally, some important questions arise from these investigations. How do strain rate (the timescale of observer) and temperature (changing the timescale of system) affect this inherent correlation between serrations and atomic rearrangements? What mechanism governs the serrations behind the spatiotemporally inhomogeneous plastic flow?

In this work, we rely on MD computer simulations to investigate the effects of strain rate and temperature on macroscopic flow serrations as well as the underlying irreversible atomic rearrangement during loading. We find that the magnitude of temporally inhomogeneous rearrangement, i.e. flow-ability determines the onset of serrated flow phenomenon. A dimensionless Deborah number is proposed to characterize the trans-scale correlation between macro-serrations and microrearrangements. 


\section{Molecular dynamic simulations}

In our MD simulations, binary $\mathrm{Cu}_{46} \mathrm{Zr}_{54}$ BMGs were used. We adopted a modified Lenard-Jones (L-J) 4-8 potential of the following form $[27,28]$ :

$\phi\left(r_{i j}\right)= \begin{cases}-\frac{A}{r_{i j}^{4}}+\frac{B}{r_{i j}^{8}}+C r_{i j}+D, & 0<r_{i j} \leqslant r_{t} \\ 0, & r_{i j}>r_{t}\end{cases}$

where $r_{i j}$ is the distance between the atoms $i$ and $j, A, B, C$ and D are constants whose values can be found in Ref. [28], $r_{t}$ is the truncation distance with the values of 5.08, 5.58 and $6.00 \AA$ for $\mathrm{Cu}-\mathrm{Cu}, \mathrm{Cu}-\mathrm{Zr}$ and $\mathrm{Zr}-\mathrm{Zr}$ pairs, respectively. The motion of each atom was evaluated by integrating the Newtonian equations of motion using velocity-Verlet method with a time step of $1 \mathrm{fs}$. To obtain glassy structures, the melt-quench procedure was used. We first placed randomly all atoms, with initial velocities of zero, into a face-centered cubic (fcc) crystal lattice in a supercell, where periodic boundary condition (PBC) was applied in all three dimensions, and then heated it to $2400 \mathrm{~K}$. After sufficiently melting, we cooled it down to $1 \mathrm{~K}$ and $500 \mathrm{~K}$, respectively, by rapid quench of cooling rate of $25 \mathrm{~K} / \mathrm{ps}$. The glass transition temperature $T_{\mathrm{g}}$ was estimated as $700 \mathrm{~K}$. Thus, we obtained two initial glassy structures with different initial temperatures ( $1 \mathrm{~K}$ and $500 \mathrm{~K}$ ). To allow them to find a local energy minimum with near-zero stress components, we repeatedly adjusted their dimensions, and reposition each atom accordingly. In this process, the NPT ensemble was used. Finally, both structures were guided toward a low-stress state (all stress components less than $10 \mathrm{MPa}$ ). Then for the subsequent indentation simulations, we set the top boundary free and fixed a layer of $6.0 \AA$ in thickness at the bottom. Another 100 ps was carried out to a new equilibration. Now, we have two three-dimensional samples that contain 432,000 atoms: Sample I with the size of $250 \times 250 \times 125 \AA^{3}$ and initial temperature of $1 \mathrm{~K}$; Sample II with the size of $260 \times 260 \times 127 \AA^{3}$ and its initial temperature of $500 \mathrm{~K}$. The amorphous structures were confirmed by their respective radial distribution functions (RDF).

A spherical indenter without atomic nature was used in the nanoindentation simulations. The indenter was modeled by a purely repulsive potential with the form $[29,30]$

$V(r)=E \cdot(R-r)^{3} \cdot \theta(R-r)$

where $r$ is the distance from the indenter center to a sample atom, $R$ is the radius of the indenter which is chosen as $20 \AA$ for all samples, $\theta(R-r)$ is the standard step function, and $E$ is a constant which is equal to 3.0 and $3.9 \mathrm{nN} / \AA^{2}$ for the tip meets a $\mathrm{Cu}$ atom and a $\mathrm{Zr}$ atom, respectively [30]. The indenter was displaced towards the top surface of the sample at a constant strain rate by keeping an invariable displacement interval of $0.1 \AA$ and adjusting the relaxation time for each displacement interval. In this process, the control to the temperature ( $1 \mathrm{~K}$ or $500 \mathrm{~K}$ ) was only allowed in a layer of $10.0 \AA$ in thickness which is just above the fixed layer at the bottom. For sample I, the total indentation depth was $15 \AA$, and three strain rates, $10^{11}, 10^{10}$ and $10^{9} \mathrm{~s}^{-1}$, were executed; for sample II, the same depth and only medium-strain rate of $10^{10}$ were performed. Parallel computing was used in all the simulation processes.

\section{Results and discussion}

Fig. 1 shows the $\mathrm{p}-\mathrm{h}$ relationships (upper curves) for the indentation simulations on sample I at three strain rates. It can be clearly seen that the serrated plastic flow is strongly rate-dependent. When the strain rate decreases from $1 \mathrm{a}$ to $1 \mathrm{c}$, the serrations become more obvious. The simulation result agrees well with a series
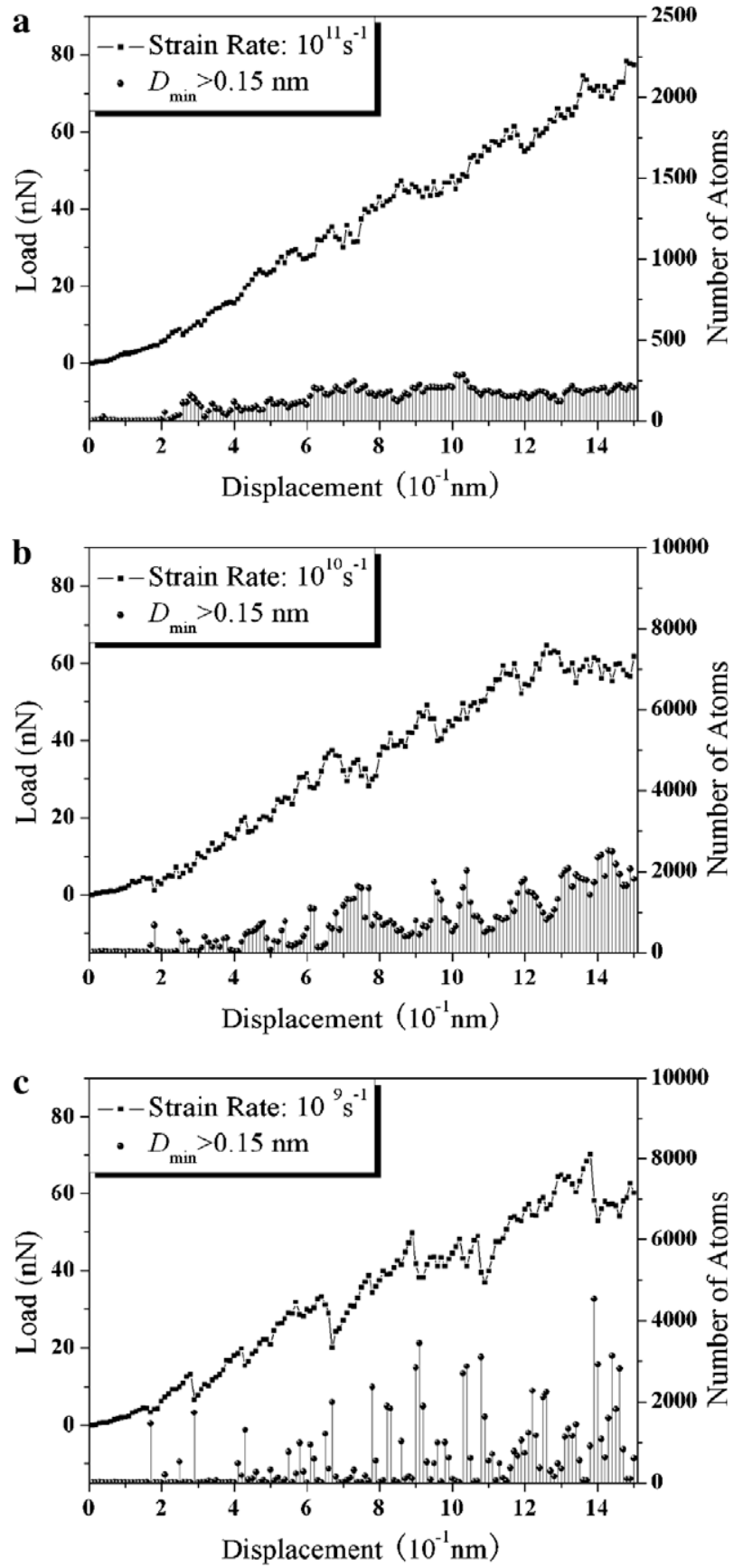

Fig. 1. The load-displacement curves (upper) and the corresponding number (lower sphere-bar) of rearranged atoms at various strain rates: (a) $10^{11} \mathrm{~s}^{-1}$, (b) $10^{10} \mathrm{~s}^{-1}$, (c) $10^{9} \mathrm{~s}^{-1}$, for sample I.

of experimental observations for real BMGs under nanoindentation [10-19]. It has been recognized that the plastic flow occurs as a result of a number of irreversible atomic rearrangements [3-6]. Thus, we use the parameter $D_{\min }$, introduced by Shi and Falk [5], to identify such rearrangement. $D_{\text {min }}$ values of all atoms during each displacement interval $(0.1 \AA)$ were calculated. We selected $1.5 \AA$, about half of the average distance between a $\mathrm{Cu}$ atom and $\mathrm{ar}$ atom in samples [5,31], as a cutoff of $D_{\min }$ to characterize the rearrangements that make up a plastic event at all strain rates. It is important to point out that the method of choosing the cutoff may be a little rough, considering its value may be affected by stain rate and temperature; nevertheless, it is efficient to judge the plastic deformation, and should not significantly change the trend. Any 
atom with larger likelihood of motion must be of greater probability of irreversible rearrangement. The numbers of the rearranged atoms with $D_{\min }>1.5 \AA$ at all intervals are displayed in Fig. 1 as black spheres under $\mathrm{p}-\mathrm{h}$ curves. We find that the atomic rearrangements are also dependent on strain rates. When strain rate decreases, the number of the rearranged atoms is larger, whereas their distribution becomes more inhomogeneous temporally. Moreover, a strong correlation of such numbers of the rearranged atoms with serrations in the p-h curves is recovered, just as Shi and Falk found [25]. The more obvious the flow serration is, the larger the number of the rearranged atoms in that interval is. The trend is more pronounced with decreasing strain rates. The spatial configurations of atomic rearrangements during a displacement interval under the three strain rates are shown in Fig. 2, where spheres with large radius represent $\mathrm{Zr}$-atoms; ones with small radius are $\mathrm{Cu}$-atoms. The hotter the atoms color, the larger their $D_{\min }$ values. It is noted that at high strain rate, few atoms rearrange, and while many rearrange at low rates. The BMG material exhibits different flow-ability under various strain rates. Better flow-ability produces more significant serrations. The high-temperature sample's $\mathrm{p}-\mathrm{h}$ curve is shown in Fig. 3a, in which the corresponding atomic rearrangements are also displayed as black spheres. Com-
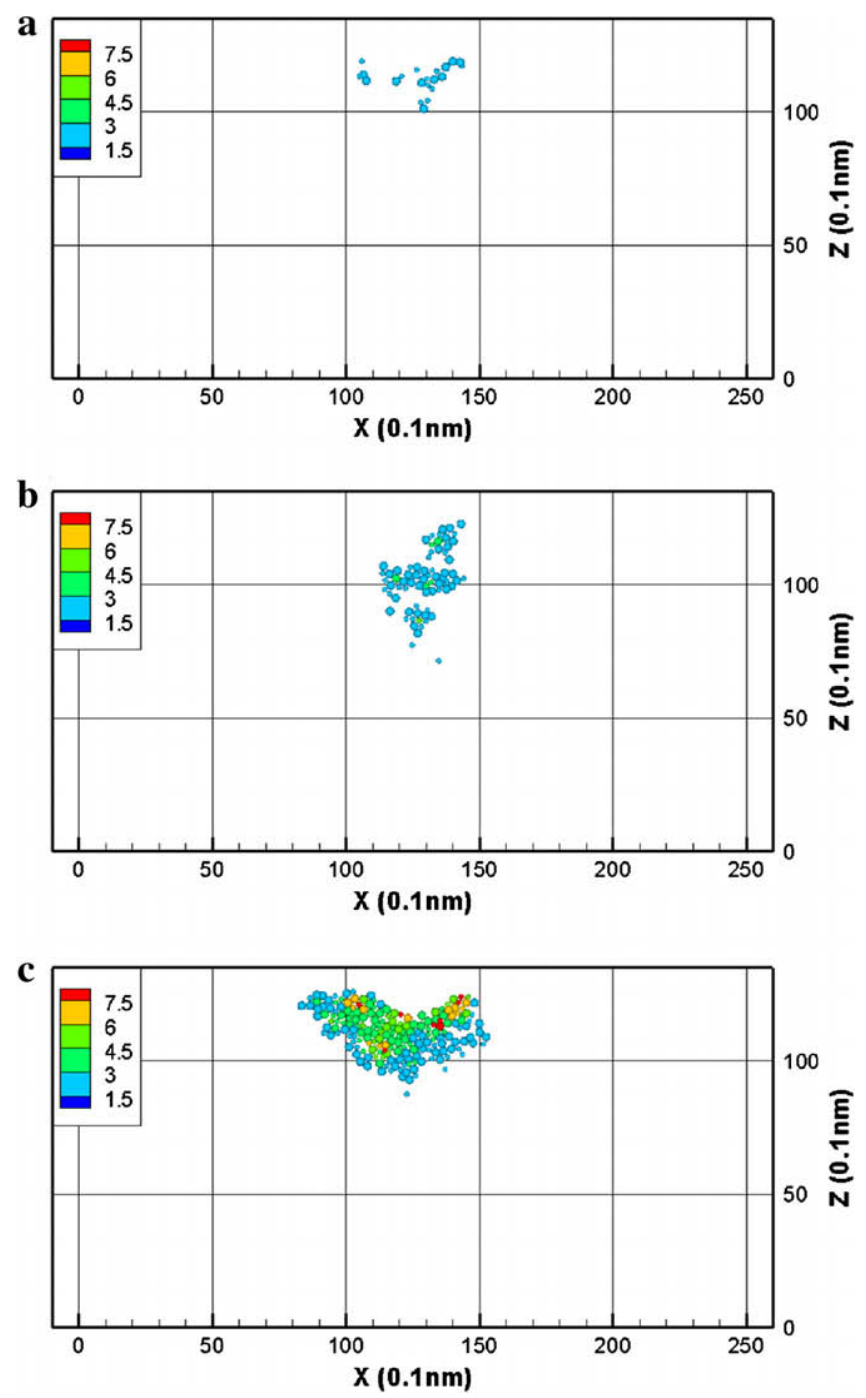

Fig. 2. The spatial distributions of the rearranged atoms with $D_{\min }>1.5 \AA$ in a displacement interval at various strain rates: (a) $10^{11} \mathrm{~s}^{-1}$, (b) $10^{10} \mathrm{~s}^{-1}$, (c) $10^{9} \mathrm{~s}^{-1}$, for sample I.
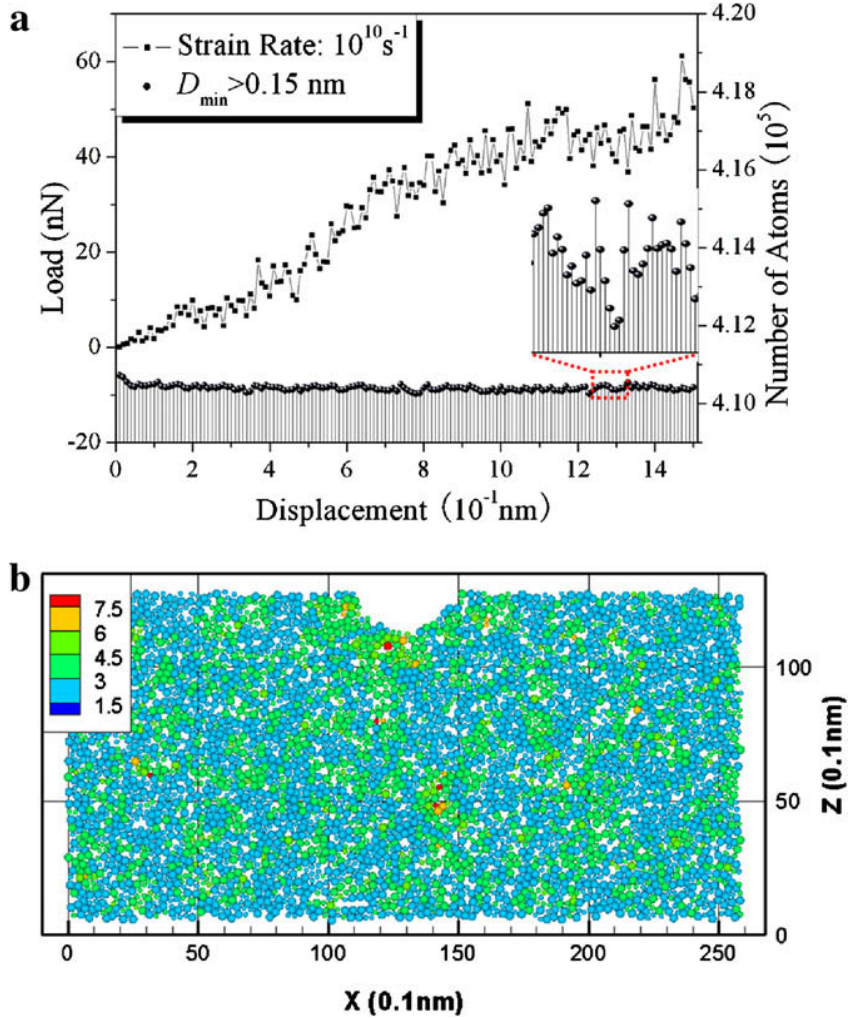

Fig. 3. The nanoindentation of sample II with the temperature of $500 \mathrm{~K}$. (a) The load-displacement curves (upper) and the corresponding number (lower spherebar) of rearranged atoms at the strain rate of $10^{10} \mathrm{~s}^{-1}$, (b) the spatial configuration of rearranged atoms in a displacement interval.

pared to Fig. 1b, we find that high-temperature results in more distinct serrations, although atomic rearrangements are superficially "homogenous" temporally. Actually, almost all of atoms within sample participate in rearrangement, leading to spatially homogeneous deformation during individual interval, as shown in Fig. $3 \mathrm{~b}$. Therefore, a fraction of number fluctuation of these rearranged atoms can bring out significant serrations, because the absolute numbers of rearranged atoms at higher temperature are quite larger than that at relatively lower temperature, not at the same order of magnitude at all. In fact, although the flow is spatially homogeneous at high-temperature, it is still temporally inhomogeneous (see the inset to Fig. 3a). In such case, the serrated flow, resulting from good flow-ability, is more obvious than that in the low-temperature case. For example, during the steady-state creep experiments on Pd-based BMGs under high temperature (>550 K) and low stress, serrations are also widely observed, even if the flow is homogeneous (no shear-banding) [32]. Thus, the BMG's flow-ability, characterized by the numbers of rearranged atoms, is very important to its serrations. From the above results, one can conclude that serrated plastic flow is mainly determined by the magnitude of its underlying irreversible atomic rearrangements that must be temporally inhomogeneous.

The serrated flow is essentially a trans-scale, connecting macroflow with micro-atomic-rearrangement, and (strain) rate-dependent process. How to characterize this phenomenon is a key point. In the field of science of rheology, the so-called Deborah number plays an important role, since it describes the influence of timescale on the observed flow behaviors [33]. Recently, Bai et al. [34] have demonstrated that the Deborah number is very important to understand the micro-damage evolution effect on macroscopic properties, which is also a rate-dependent tarns-scale problem [35]. The flow of BMGs due to its viscosity in nature can 
be characterized by a dimensionless Deborah number that is the ratio between the timescale of the phenomenon and that of the observation method [36]. In the present case, we define Deborah number as

$D e=\frac{t_{r}}{t_{e}}$

where $t_{r}$ is the Maxwell time or internal structural relaxation time under loading, and $t_{e}$ is the macroscopic imposed time of external loading. The magnitude of the Deborah number provides interesting indications. If $t_{r}$ is very short compared with $t_{e}$, i.e. De $<<1$, we see metallic glasses behaving as an ordinary viscous fluid. However, in the opposite case, i.e. De >> 1, they behave as a glassy solid. Obviously, changing strain rates modifies the timescale of the observer, i.e. $t_{e}$, while the change in temperature influences the timescale of system, i.e. $t_{r}$ [37]. According to the MD simulation results, we find that decreasing strain rates or elevating temperature brings out a good flow-ability, implying a smaller Deborah number; hence, producing more prominent serrations. In order to highlight the essential physical argument, we consider a thermomechanical deformation of a BMG undergoing one-dimensional simple homogeneous shearing [38] to examine the strain rate and temperature effects on Deborah number. The governing equations for this problem can be written as

$\dot{\gamma}=\frac{\dot{\tau}}{G}+2 f \exp \left(-\frac{\Delta G^{m}}{k_{B} \theta}\right) \sinh \left(\frac{\tau \Omega}{2 k_{B} \theta}\right) \exp \left(-\frac{1}{\xi}\right)$

$\frac{\partial \theta}{\partial t}=\frac{\beta_{\mathrm{TQ}}}{\rho C_{v}} \tau \frac{\partial \dot{\gamma}^{p}}{\partial t}$

$\frac{\partial \xi}{\partial t}=g(\xi, \theta, \tau)$

where Eq. (4) states that the total strain-rate, $\dot{\gamma}$, is the sum of the elastic part (the first term on the right hand) and the plastic part $\dot{\gamma}^{p}$ (the second term on the right hand) [39]. In what follows, $\dot{\gamma}$ is re-
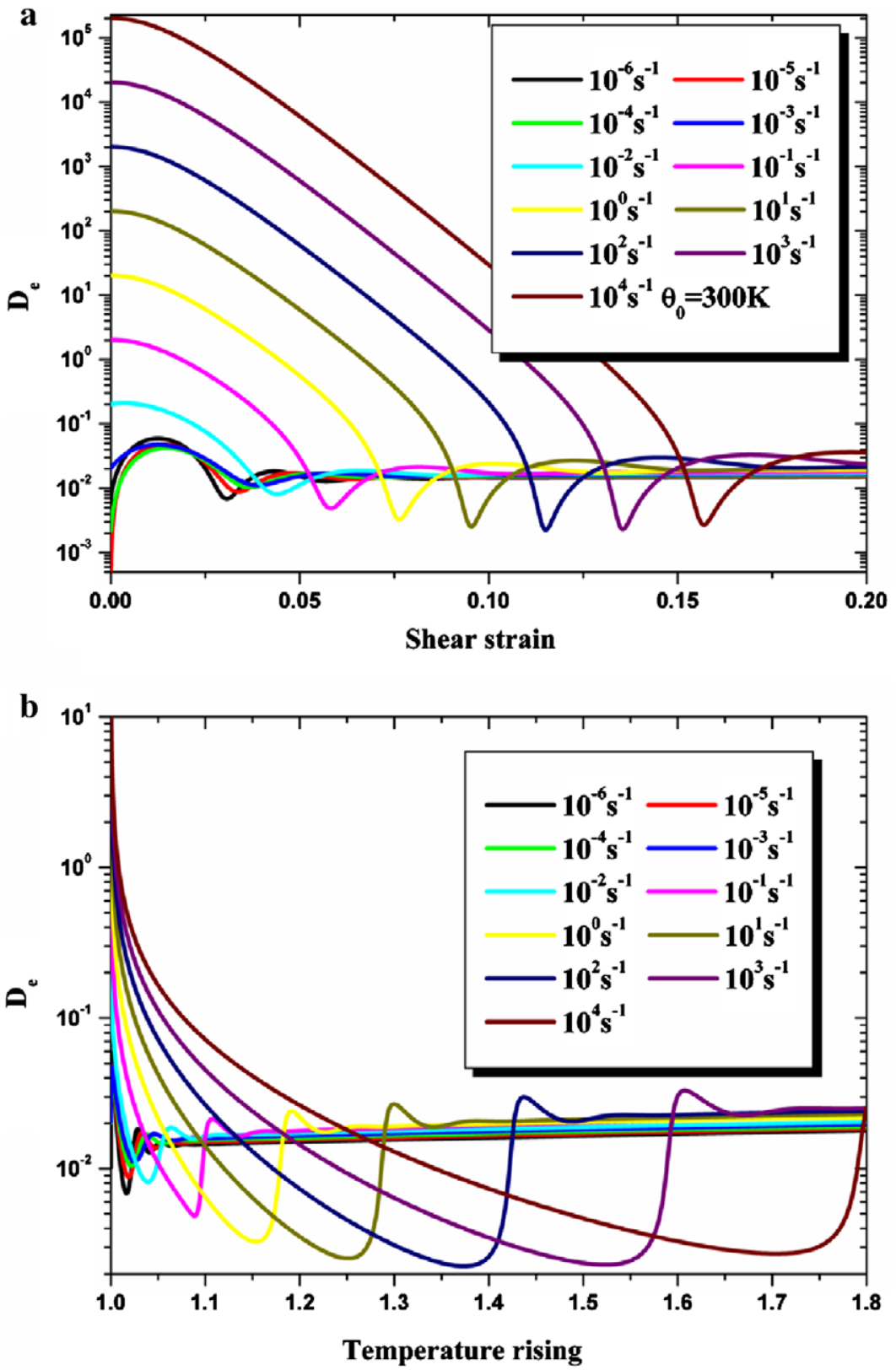

Fig. 4. (a) Calculations showing the Deborah number varies with (a) the shear strain and (b) the temperature rising under different strain rates. 
garded as a prescribed constant over the deformation history. Eq. (5) is the energy balance, and Eq. (6) describes the net rate of increase of free volume, the explicit expression of which was given by Spaepen [3]. In these equations, $\tau$ is the shear stress, $\gamma$ is the shear strain, $G$ is the shear modulus, $f$ is the frequency of atomic vibration ( $\sim$ Debye frequency), $\Delta G^{m}$ is its activation energy, $k_{\mathrm{B}}$ is the Boltzmann constant, $\Omega$ is the atomic volume, $\xi$ is the free volume concentration, $\theta$ is the temperature, $\beta_{\mathrm{TQ}}$ is the Taylor-Quinney coefficient, $\rho$ is the mass density, and $C_{\mathrm{v}}$ is the specific heat. Then, $t_{r}$ can be expressed as $t_{r}=\eta / G$ [36], where the viscosity $\eta=\tau / \dot{\gamma}^{p}$ [39]. $t_{e}$ can be defined as $t_{e}=\gamma / \dot{\gamma}$. By taking typical of parameters for BMGs $[36,40]$, we can numerically solve (4)-(6) for a typical range of $10^{-6} \sim 10^{4} \mathrm{~s}^{-1}$. As mentioned previously, the strain rate in our MD simulation is determined by the internal time scale of the system, the indentation displacement interval $(0.1 \mathrm{~A})$ and the indentation depth. Due to the limit of intrinsic time scale and length scale in the MD method, the strain rate is usually far larger than that in the continuum modeling or the real experiment. How to correlate exactly the strain rates in the two kinds of modes is beyond the scope of our present study. However, the following calculation results about the Deborah number can help readers to understand the MD results, because the effect of strain rates on the plastic flow should show a similar tendency in the two modes. The variations of Deborah number with shear strain at different strain rates and the initial temperature $\theta_{0}=300 \mathrm{~K}$ are shown in Fig. $4 \mathrm{a}$. From the figure, one can find that when $\dot{\gamma}<10^{-2} \mathrm{~s}^{-1}$, the Deborah number is far less than unit during the whole deformation history. This indicates that BMGs are of perfect flow-ability under relatively low strain rates. In addition, we note that in this range, the BMG's flow is insensitive to strain rates. Once $\dot{\gamma}>10^{-2} \mathrm{~s}^{-1}$, the flow behavior exhibits strongly strain rate dependence. The values (far more than unit) of Deborah number at high-strain rates are larger than those at low-strain rates in the early deformation stage. The high-strain rate decreases the flow-ability, via decreasing $t_{e}$. This is the main reason that the serrations are milder at higher strain rates, which is confirmed by our MD simulations. Interestingly, we find that the rate-dependent critical point is $\dot{\gamma}=10^{-2} \mathrm{~s}^{-1}$, i.e. $t_{e} \sim 100 \mathrm{~s}$. This value is just consistent with the structural relaxation time at $T_{\mathrm{g}}$ [41]. Hence, the onset criterion for serrated flow in BMgs can be proposed as De $<1$. Fig. $4 \mathrm{~b}$ displays the temperature rising influence on the Deborah number under various strain rates. It can be seen that the Deborah number decreases with the rising $\left(\theta / \theta_{0}\right)$ in temperature, prior to a steadystate flow. This means that high-temperature promotes the serrated plastic flow through decreasing the structural relaxation time [37]. This result is consistent with the available experimental observations $[14,32,42]$ and our present MD simulations.

\section{Conclusion}

Spherical nanoindentations on a model $\mathrm{Cu}_{46} \mathrm{Zr}_{54}$ metallic glass, containing 432,000 atoms, with different initial temperature ( $1 \mathrm{~K}$ and $500 \mathrm{~K}$ ), were conducted by using molecular dynamics simulations. The strain-rate and temperature dependent serrated plastic flow is observed; decreasing strain rate or increasing temperature results in more prominent serrations. Furthermore, the irreversible atomic rearrangement underpinning the serrated flow was probed. We find that the onset of serrated flow is slaved by the magnitude of such rearrangement, i.e. flow-ability that is temporally inhomo- geneous. A dimensionless Deborah number for this trans-scale and rate-dependent phenomenon is introduced to characterize the effects of strain rate and temperature on serrations. The calculation results show that the obvious serrated plastic flow occurs when the Deborah number is smaller than unit. Our findings are well consistent with the available experimental observations and other simulations.

\section{Acknowledgements}

Financial support from the Natural Science Foundation of China (Grants Nos. 10725211, 10721202, 10872206), the Major State Basic Research Development Program of China (973 program) (Grant No. 2009CB724401) and the Key Project of Chinese Academy of Sciences (Nos. KJCX-SW-L08 and KJCX2-YW-M04) is acknowledged. All computing of this work was carried out by Supercomputer DeepComp 6800, and we thank Dr. Yangde Feng of Super Computing Center of Chinese Academy of Science for his help in computations.

\section{References}

[1] J.D. Bernal, Nature 185 (1960) 68.

[2] H.W. Sheng, W.K. Luo, F.M. Alamgir, J.M. Bai, E. Ma, Nature 439 (2006) 419.

[3] F. Spaepen, Acta Metall. 25 (1977) 407.

[4] A.S. Argon, Acta Metall. 27 (1979) 47.

[5] M.L. Falk, J.S. Langer, Phys. Rev. E 57 (1998) 7192.

[6] M.L. Falk, Science 318 (2008) 1880.

[7] W.L. Johnson, Mater. Res. Soc. Bull. 24 (1999) 42.

[8] W.H. Wang, C. Dong, C.H. Shek, Mater. Sci. Eng. R 44 (2004) 45.

[9] M.F. Ashby, A.L. Greer, Scr. Mater. 54 (2006) 321.

[10] W.J. Wright, R. Saha, W.D. Nix, Mater. Trans. JIM 42 (2001) 642.

[11] W.H. Jiang, M. Atzmon, J. Mater. Res. 18 (2003) 755.

[12] C.A. Schuh, A.S. Argon, T.G. Nieh, J. Wadsworth, Phil. Mag. 83 (2003) 2585

[13] C.A. Schuh, T.G. Nieh, Acta Mater. 51 (2003) 87

[14] C.A. Schuh, A.C. Lund, T.G. Nieh, Acta Mater. 52 (2004) 5879.

[15] L.H. Dai, L.F. Liu, M. Yan, B.C. Wei, J. Eckert, Chin. Phys. Lett. 21 (2004) 1593.

[16] A.L. Greer, A. Castellero, S.V. Madge, I.T. Walker, J.R. Wilde, Mater. Sci. Eng. A 375 (2004) 1182

[17] G.P. Zhang, W. Wang, B. Zhang, J. Tan, C.S. Liu, Scr. Mater. 52 (2005) 1147.

[18] J.-I. Jang, B.G. Yoo, J.Y. Kim, Appl. Phys. Lett. 90 (2007) 211906.

[19] B. Yang, T.G. Nieh, Acta Mater. 55 (2007) 295.

[20] W.H. Li, B.C. Wei, T.H. Zhang, D.M. Xing, L.C. Zhang, Y.R. Wang, Intermetallics 15 (2007) 706.

[21] W.H. Jiang, G.J. Fan, F.X. Liu, G.Y. Wang, H. Choo, P.K. Liaw, Int. J. Plasticity 24 (2007) 1.

[22] Y. Zhang, A.L. Greer, Appl. Phys. Lett. 89 (2006) 071907

[23] J.J. Lewandowski, A.L. Greer, Nat. Mater. 5 (2006) 15.

[24] Y. Shi, M.L. Falk, Appl. Phys. Lett. 86 (2005) 011914.

[25] Y. Shi, M.L. Falk, Thin Solid Films 515 (2007) 3179.

[26] Y. Shi, M.L. Falk, Acta Mater. 55 (2007) 4317.

[27] S. Kobayashi, K. Maeda, S. Takeuchi, J. Phys. Soc. Jpn. 48 (1980) 1147.

[28] A.C. Lund, C.A. Schuh, Acta Mater. 51 (2003) 5399.

[29] C.L. Kelchner, S.J. Plimpton, J.C. Hamilton, Phys. Rev. B 58 (1998) 11085

[30] X.L. Ma, W. Yang, Nanotechnology 14 (2003) 1208.

[31] A. Gannepalli, S.K. Mallapragada, Nanotechnology 12 (2001) 250.

[32] M. Heggen, F. Spaepen, M. Feuerbacher, J. Appl. Phys. 97 (2005) 033506.

[33] M. Reiner, Phys. Today 17 (1964) 62.

[34] Y.L. Bai, M.F. Xia, H.Y. Wang, F.J. Ke, Particuology 1 (2003) 7.

[35] Y.L. Bai, H.Y. Wang, M.F. Xia, F.J. Ke, Acta Mech. Sin. 24 (2008) 111

[36] L.F. Liu, L.H. Dai, Y.L. Bai, B.C. Wei, J. Non-Cryst. Solids 351 (2005) 3259.

[37] G. Adam, J. Bibbs, J. Chem. Phys. 43 (1965) 139.

[38] L.H. Dai, M. Yan, L.F. Liu, Y.L. Bai, Appl. Phys. Lett. 87 (2005) 141916.

[39] P.S. Steif, F. Spaepen, J.W. Hutchinson, Acta Metall. 30 (1982) 447.

[40] Y.F. Gao, B. Yang, T.G. Neih, Acta Mater. 55 (2007) 2319.

[41] T. Scopigno, G. Ruocco, F. Sette, G. Monaco, Science 302 (2003) 849

[42] J. Lu, G. Ravichandran, W.L. Johnson, Acta Mater. 51 (2003) 3429. 